\title{
Estágio Curricular Supervisionado I: a atuação do acadêmico de Enfermagem na realidade da ESF em tempos de pandemia
}

\author{
Supervised Curriculum Internship I: Nursing academic performance in the reality of the ESF in
}

times of pandemic

Práctica Currícula Supervisada I: desempeño académico de Enfermería en la realidad de la ESF en tiempos de pandemia

Brenda da Rocha Carvalho

ORCID: https://orcid.org/0000-0001-6611-9766 Faculdade Irecê, Brasil

E-mail: brendauibai@outlook.com

Taíse Rocha Santos

ORCID: https://orcid.org/0000-0002-7895-8409 Faculdade Irecê, Brasil

E-mail: taysesrocha@hotmail.com

Liliane Machado Ferreira

ORCID: https://orcid.org/0000-0001-8837-2332 Faculdade Irecê, Brasil

E-mail: lilianejlili@ hotmail.com

Gisele Gomes Dourado

ORCID: https://orcid.org/0000-0003-3282-4015 Faculdade Irecê, Brasil

E-mail: gisele97dourado@gmail.com

Giovana Alecrim Rocha

ORCID: https://orcid.org/0000-0003-2878-9800 Faculdade Irecê, Brasil

E-mail: giovana0406rocha@gmail.com

Nandiara Nunes Vieira

ORCID: https://orcid.org/0000-0002-8281-1506 Faculdade Irecê, Brasil

E-mail: nandiara9@hotmail.com

Afonso Nunes Marques

ORCID: https://orcid.org/0000-0003-0055-9624 Faculdade Irecê, Brasil

E-mail:afonso-nunes07@hotmail.com Iago Araújo Duarte

ORCID: https://orcid.org/0000-0002-8192-6977 Faculdade Irecê, Brasil

E-mail: Iago23araujo@gmail.com

Eveline Souza da Silva

ORCID: https://orcid.org/0000-0003-1957-6619 Faculdade Irecê, Brasil

E-mail: eveline95souza@gmail.com

Adriano da Silva Borges

ORCID: https://orcid.org/0000-0002-4706-9457 Faculdade Irecê, Brasil

E-mail: Adrianoborges1420@gmail.com

\begin{abstract}
Resumo
Objetivo: relatar vivência do acadêmico de enfermagem na realidade da ESF em tempos de pandemia durante o Estágio Supervisionado I. Metodologia: Trata-se de um relato de experiência ocorrido em duas ESF no interior da Bahia, por uma acadêmica de enfermagem do $9^{\circ}$ semestre. Essa atividade foi desenvolvida de 08 de fevereiro de 2021 a 06 de julho de 2021, correspondendo ao período letivo 2021.1 da faculdade. Resultados e discussão: A rotina de atendimentos na ESF enfrenta grandes desafios devido à pandemia. As pessoas com Doenças Crônicas Não Transmissíveis (DCNT) precisam de atenção especial nesse contexto, pois apresentam duplo risco em seu estado de saúde. Os profissionais de saúde passam por situações que abalam a saúde mental dos mesmos, devido à sobrecarga e conflitos frente a situações que fogem do controle da ESF. Considerações finais: Durante a pandemia o sentimento de medo e insegurança muitas vezes se fizeram presentes, mas serviram para fortalecer os futuros enfermeiros.
\end{abstract}


Palavras-chave: Estratégia Saúde da Família; Covid-19; Estudantes de enfermagem.

\begin{abstract}
Objective: to report the experience of the nursing student in the reality of the FHS in times of pandemic during Supervised Internship I. Methodology: This is an experience report that took place in two FHS in the countryside of Bahia, by a nursing student from the 9th semester. This activity was carried out from February 8, 2021 to July 6, 2021, corresponding to the academic term 2021.1 of the faculty. Results and discussion: The routine of care in the ESF faces major challenges due to the pandemic. People with Chronic Non-Communicable Diseases (NCDs) need special attention in this context, as they present a double risk in their health status. Health professionals go through situations that affect their mental health, due to overload and conflicts in situations that are beyond the control of the ESF. Final considerations: During the pandemic, the feeling of fear and insecurity was often present, but they served to strengthen future nurses.
\end{abstract}

Keywords: Family Health Strategy; Covid-19; Nursing students.

\title{
Resumen
}

Objetivo: reportar la experiencia del estudiante de enfermería en la realidad de la ESF en tiempos de pandemia durante el Internado Supervisado I. Metodología: Se trata de un relato de experiencia que se llevó a cabo en dos ESF del interior de Bahía, por un estudiante de enfermería del $9^{\circ}$ semestre. Esta actividad se llevó a cabo del 8 de febrero de 2021 al 6 de julio de 2021, correspondiente al semestre académico 2021.1 de la facultad. Resultados y discusión: La rutina de atención en la ESF enfrenta grandes desafíos debido a la pandemia. Las personas con Enfermedades Crónicas No Transmisibles (ENT) necesitan una atención especial en este contexto, ya que presentan un doble riesgo en su estado de salud. Los profesionales de la salud atraviesan situaciones que afectan su salud mental, por sobrecarga y conflictos en situaciones que escapan al control de la ESF. Consideraciones finales: Durante la pandemia, el sentimiento de miedo e inseguridad a menudo estuvo presente, pero sirvió para fortalecer a las futuras enfermeras.

Palabras clave: Estrategia de Salud de la Familia; Covid-19; Estudiantes de enfermería.

\section{Introdução}

O Estágio Curricular Supervisionado I faz parte da grade de formação dos enfermeiros, com a finalidade de criar vínculo entre os acadêmicos e a população, bem como propiciar a vivência em um campo de prática tão vasto quanto a Estratégia de Saúde da Família (ESF). Devido à pandemia da Covid-19, esse estágio passou por reformulações e cuidados, tanto para o bem-estar dos alunos quanto da população assistida.

A pandemia está causando sofrimento a nível mundial, devido à sua gravidade e magnitude. A covid-19 é uma infecção viral, causada pelo vírus SARS-cov2, transmitida por meio de aerossóis, gotículas, secreções respiratórias e contato direto, que atinge principalmente as vias aéreas inferiores, podendo progredir para um quadro de Insuficiência Respiratória Aguda Grave (SARS) e complicações a nível pulmonar e cardiovascular (Tesini, 2021). Em casos leves há sinais e sintomas como febre, tosse, mialgia, astenia, dispneia leve, ageusia e anosmia, já em casos complicados há agravamento do quadro clínico, sendo necessário atendimento hospitalar. Assim, percebe-se que essa infecção afetou todos os indivíduos e setores, sobretudo os serviços de saúde (Cunha et al., 2020).

A ESF é o principal modelo de Atenção Primária à Saúde (APS) em consonância com os princípios do Sistema Único de Saúde (SUS): universalidade, integralidade e equidade. A importância dela é evidenciada pela cobertura de aproximadamente 131 milhões de brasileiros, agindo através da orientação comunitária, que faz a prevenção, vigilância, rastreamento e acompanhamento de casos, assim como a identificação de contatos e população vulnerável (Giovanella et al., 2021).

Assim, fica explícito o quanto está sendo necessário adaptações em todos os serviços para lidar com a pandemia. A reorganização da ESF se fez também necessária para continuar cuidando da saúde dos cidadãos de maneira integral. Além dos cuidados que já eram ofertados antes da pandemia, houve o acréscimo da atuação em casos de covid-19, trabalhando com teleatendimento, prevenção, identificação de casos suspeitos, estratificação da doença, manejo terapêutico e monitoramento do isolamento domiciliar em casos leves (Brasil, 2020a). 
Desse modo o componente curricular tem por finalidade inserir o estudante no contexto da APS, conhecer a territorialização dos locais e realidade diária das ESF, desde a gestão até a assistência direta aos pacientes, trabalhando a capacidade de comunicação e de resolução de problemas dos estudantes, permitindo ainda o aprimoramento de técnicas.

Enfatiza-se ainda o propósito de ambientar o acadêmico na equipe multidisciplinar da ESF sobretudo compreendendo esse funcionamento no período de pandemia. Diante do impacto na saúde nesse momento, destaca-se a importância da atuação de uma equipe multiprofissional nos ambientes de saúde, pois é um cenário desafiador, sendo possível a obtenção de resultados satisfatórios com o trabalho em equipe pautado em saberes científicos, intervindo nas diversas áreas, olhando o paciente como um todo (Silva, Silva, Santos, \& Ferreira, 2021). O trabalho interdisciplinar é destacado durante toda a graduação, de modo a culminar em profissionais conscientes e capacitados.

Destarte, esse trabalho se justifica devido ao momento de estágio na ESF ainda em momento crítico da pandemia, sendo possível observar desde adaptações na logística quanto nos profissionais, gerando reflexões sobre os riscos enfrentados e o bem-estar dos funcionários e da população. Acredita-se na sua relevância para a pesquisa ao trazer experiências em um momento crítico a nível mundial. Dessa forma, esse estudo tem por objetivo: relatar vivência do acadêmico de enfermagem na realidade da ESF em tempos de pandemia durante o Estágio Supervisionado I.

\section{Metodologia}

Trata-se de uma pesquisa descritiva, com abordagem qualitativa, do tipo relato de experiência. A pesquisa descritiva se configura em detalhar as características de determinados grupos ou populações, se aliando ao pressuposto da abordagem qualitativa que permite avaliar os dados de maneira subjetiva e profunda, mostrando a realidade da situação vivenciada (Gil, 2008).

O estudo ocorreu em duas ESF no interior da Bahia, por acadêmicos de enfermagem do $9^{\circ}$ semestre, na disciplina Estágio Supervisionado I. Essa disciplina tem o objetivo de inserir o estudante no serviço de saúde, fazendo-o atuar de acordo com a sua profissão, de maneira responsável e ética, parte do pressuposto de colocar em prática toda a teoria aprendida em semestres anteriores. Essa atividade foi desenvolvida de 08 de fevereiro de 2021 a 06 de julho de 2021, totalizando 400 horas de estágio, correspondendo ao período letivo 2021.1 da faculdade.

Os discentes ficaram divididos entre duas ESF, ambas na mesma cidade, o encontro com o supervisor/ professor acontecia na ESF, de segunda-feira à sexta-feira, respeitando o horário de funcionamento da mesma. $\mathrm{O}$ atendimento aos pacientes era realizado de maneira individual, porém o supervisor estava a todo momento disponível para retirada de dúvidas e avaliação do atendimento. Para melhor aproveitamento do estágio, em momentos que não estava tendo atendimento era realizado discussão em grupo sobre diversas temáticas.

\section{Resultados e Discussão}

Esse período de estágio dividido em duas ESF diferentes é primordial para a observação de diferenças entre os estabelecimentos, principalmente as diferenças de gestão e organização, bem como a aceitação dos acadêmicos para contribuir com a equipe.

As duas ESF são compostas em sua estrutura física por: uma recepção, dois banheiros, uma sala de curativos/ sutura, uma farmácia, um consultório de enfermagem, uma área para depósito de material de limpeza (DML), um consultório médico, uma triagem, uma sala de reunião, uma sala de vacina, um consultório odontológico, uma sala de observação, um expurgo, um almoxarifado, uma sala de esterilização, uma copa, uma sala de nebulização, uma Gerência, ainda contendo uma área livre que pode ser aproveitada para atividades de educação em saúde e cultivo de plantas medicinais. 
Portanto certifica- se que essas unidades estão de acordo com as normas quanto a estrutura física da Unidade Básica de Saúde (UBS) com uma ESF. Também está de acordo à Política Nacional de Atenção Básica (PNAB, 2017) onde diz que a equipe deve ser composta por: um enfermeiro, um médico, Agentes Comunitários de Saúde (ACS), uma técnica ou auxiliar de enfermagem, bem como poderá ter um dentista com uma técnica ou auxiliar de dentista. Sendo que essas unidades ainda contam com uma vacinadora.

O momento crítico da pandemia causou incertezas na equipe das ESF e nos estudantes, o contato com o paciente ainda era um impasse, pois muitos só confiavam nos profissionais de saúde da ESF. Foi notório que é de extrema importância a relação de confiança das pessoas da comunidade com a equipe da estratégia. Porém, é papel da equipe apresentar os acadêmicos de enfermagem, e incentivar os indivíduos a terem confiança nos mesmos, pois os estudantes procuram tratar cada paciente em sua individualidade e integralidade, esse apoio da equipe é essencial para o bom desenvolvimento das atividades. Na primeira ESF essa relação não foi estabelecida, houve dificuldades de aceitação dos atendimentos, mas na segunda ESF a aceitação foi perfeita, o vínculo dos acadêmicos e supervisor com a comunidade era forte. Os estudantes procuraram a todo momento seguir as regras de biossegurança nos atendimentos para o seu bem-estar e para a segurança dos pacientes.

A logística durante o período de estágio foi seguida conforme a demanda das unidades de saúde, buscando explorar ao máximo a gama de possibilidades em campo. Desse modo foi possível organizar a atuação de cada integrante, desenvolvendo o potencial de todos, aprendendo e aperfeiçoando a cada dia.

As atividades desenvolvidas e suas respectivas quantidades são: planejamento estratégico situacional- 01; consultas de saúde do homem- 0; consultas de pré-natal- 146; consultas puerperais- 32; consultas de hiperdia e avaliação de exames- 57; consultas de puericultura- 67; consultas de planejamento sexual e reprodutivo- 131; educação em saúde- 04; curativos- 93; administração de medicamentos- 208; reunião com agentes comunitários de saúde- 01; organização do fluxo na vacinação da Covid-19- 40.

Assim percebe-se que foram contempladas a vivência nos diversos programas oferecidos na ESF, além de buscar a identificação de problemas e suas respectivas soluções. As maiores produções foram administração de medicamentos, seguido por consultas de pré-natal e consultas de planejamento sexual e reprodutivo. O grande destaque foram as consultas de saúde do homem, que permaneceram zeradas até o final do estágio. Esperava-se que a produção fosse maior, mas como atenuante há o período de pandemia, em que as unidades precisaram passar por mudanças.

As regras do estágio passaram por reformulações em virtude do momento delicado vivido em todo o mundo. Foi preciso assinar um termo de responsabilidade, informando que o aluno estava ciente dos riscos, mas que optava por estagiar naquele semestre. O uso de Equipamentos de Proteção Individual (EPIs) foi reforçado, devendo o acadêmico fazer uso de: gorro, óculos de proteção ou face shield, máscara N95/PFF2, jaleco, avental para situações de maior exposição e sapato impermeável. Além disso, era realizado triagem para sinais e sintomas da Covid-19 todos os dias. A unidade de saúde também passou por adaptações, aumentando a oferta de álcool em gel para uso dos profissionais e pacientes, além de fortalecer a higienização dos locais.

Medidas de biossegurança regem os serviços de saúde nesse momento, sendo limitado o número de pessoas nos locais, organizando o fluxo para evitar aglomerações, inclusive os profissionais, evitando aglomerações em horário de descanso ou refeições, respeitando o distanciamento de um metro. O uso da máscara de tecido não é ideal para uso dos profissionais, é preconizado uso de máscara cirúrgica para contato com pacientes e máscara N95/PFF2 para realização de procedimentos que gerem aerossóis, logo, o uso de aventais e óculos de proteção também é indispensável para tais procedimentos. Reforça-se ainda a necessidade de capacitação dos profissionais para a paramentação e desparamentação de acordo às normas, pois essa ainda não é uma realidade positiva nos estabelecimentos de saúde (Agência Nacional de Vigilância Sanitária [Anvisa], 2021). 
Ratifica-se a importância do uso de EPIs todo o tempo, para proteção dos profissionais, pacientes e acadêmicos. Logo, foi respeitado todas a normas, inclusive houve a capacitação de todos os estudantes que iriam para campo de estágio, a fim de esclarecê-los sobre normas de precaução, ressaltando o devido distanciamento, uso de EPIs e cuidados durante a paramentação e desparamentação, de forma a evitar o contágio pelo vírus SARS- cov2. Além do mais foi passado orientações quanto o aviso de qualquer sintoma respiratório, com a finalidade de afastamento do campo de estágio, e posterior análise com teste.

As medidas de biossegurança têm por finalidade o controle e a prevenção de infecções, culminando na proteção e segurança dos profissionais, clientes e acompanhantes, não devendo assim serem negligenciados. A utilização de EPIs e a higienização das mãos são pilares essenciais para a proteção de todos, cumprindo medidas específicas para interromper a barreira de transmissão em todos os setores (Cavalcante, Pereira, Leite, Santos, \& Cavalcante, 2019).

Durante essa pandemia fica explícito a importância da superação do senso comum na sociedade, pois essa superação é possível apenas através do posicionamento crítico, da relação ensino- aprendizagem. Os cidadãos através de seus professores passam pela transferência de ideias, porém é preciso ir além para buscar resolução de dilemas sociais como esse enfrentado atualmente, enfatizando que o conhecimento, diálogo e respeito são imprescindíveis para o enfrentamento e mudança da realidade, reestruturando o processo de ensino, formando indivíduos críticos (Marques \& Fraguas, 2021).

A rotina de atendimentos na ESF enfrenta grandes desafios devido à pandemia, principalmente por trabalhar com programa continuado e programado, promoção da saúde e prevenção de agravos, portanto, são cuidados essenciais, porém, não são de carácter emergencial. Assim terminou por gerar diminuição dos atendimentos no início, e logo após foram pensadas estratégias para o restabelecimento das funções da ESF.

É importante salientar que além das habilidades assistenciais desenvolvidas durante o período de estágio, possui ainda as competências gerenciais que se fazem presente na forma de liderança, comunicação, capacidade de resolução de problemas, administração e gerenciamento do serviço (Rigobello et al., 2018). Essas competências são vivenciadas nas práticas, durante o estágio através do Planejamento Estratégico Situacional (PES), que propõe aos acadêmicos o desafio de conhecer o local em toda a sua dimensão e elencar os principais problemas possíveis, com capacidade de resolução e trazer bons resultados.

Durante a apresentação do cronograma do estágio, foi proposto a definição de um líder/gerente em cada semana. Desse modo, esse seria o responsável por desenvolver a escala semanal, atribuindo a cada discente um setor ou atividade, estando com esse gerente a responsabilidade de supervisão, gerenciamento e resolução de problemas dentro das suas limitações. Essa particularidade permite que o acadêmico desenvolva capacidade gerencial que se torna tão relevante ao profissional de enfermagem atuante no mercado de trabalho.

Levando em consideração a atuação do enfermeiro da ESF, no contexto da pandemia, foi possível observar a dimensão e importância da sua atuação como gestor. Nesse período, além de toda exposição, esses profissionais foram responsáveis pela reorganização do processo de trabalho, gerindo recursos humanos, materiais e financeiros, mantendo a oferta dos serviços de saúde dentro das unidades, mesmo diante de todas as limitações.

Dessa forma, os profissionais de saúde, sobretudo os enfermeiros precisaram fazer o planejamento estratégico das situações, sendo ainda de extrema importância que os profissionais conheçam a sintomatologia da Covid-19 para conseguir intervir antecipadamente nos casos e fornecer orientações quanto a prevenção dela. Vale ressaltar a necessidade de reorganização do fluxo, acompanhamento virtual e encaminhamento precoce para preservar a saúde da gestante e do feto, estabelecendo um plano de cuidados especial para esse grupo (Estrela, Silva, Cruz, \& Gomes, 2020).

Houve a utilização do PES, que consiste em uma ferramenta de gestão, sendo desenvolvido pelos discentes em conformidade com o supervisor, a fim de identificar problemas e agir diante dos mesmos, estabelecendo medidas. Assim trabalhou-se a apreciação situacional com abordagem participativa, englobando todos os atores envolvidos, ou seja, a equipe multiprofissional, valorizando as contribuições de cada indivíduo. Portanto o PES engloba a detecção de nós críticos que são 
passíveis de intervenção prática, o estabelecimento de objetivos, o planejamento de intervenções, a implementação destas e a avaliação (Lima \& Lima, 2020).

Dentre todas as fragilidades de atendimento identificadas, foi trabalhado a ausência dos homens na ESF, pois eles comparecem apenas para vacinação da Covid-19, mas não aderem aos programas de acompanhamento e prevenção. Como forma de intervenção, realizou-se uma conversa com os homens no dia da vacinação, sobre qual sua rotina de procura à ESF e se considerava o atendimento importante, e se não frequentava, qual o motivo pela não procura do serviço, além do diálogo com eles sobre as principais patologias que acometem o público masculino, sobretudo as Infecções Sexualmente Transmissíveis (ISTs) e como a ESF pode ajudar. Após essas conversas com os homens, houve o diálogo com os ACS, os incentivando a convidarem os homens a comparecer à unidade.

Essa educação em saúde com os homens é de extrema relevância, devido a vulnerabilidade deles quanto à saúde, sobretudo as ISTs, enfatizando a Tricomoníase. Portanto, ratifica-se a necessidade de incentivo a práticas de educação em saúde voltadas para a saúde sexual e reprodutiva, abordando o uso de preservativos, responsabilidade com a saúde, culminando na saúde sexual e reprodutiva, que o planejamento não é apenas das mulheres, e sim uma responsabilidade mútua (Cruz et al., 2021).

Ao saber que as gestantes são grupo de risco e que seu acompanhamento deve ser feito prioritariamente na ESF, foi necessário diminuir a quantidade de grávidas por dia para consulta de pré-natal, evitando aglomeração, bem como foi aumentado a oferta de álcool gel e álcool a 70\% para elas. Ainda foi orientado que elas não saíssem de casa, salvo em momentos que fossem extremamente necessários, orientando também sobre o momento do parto que provavelmente seria no hospital de referência, incentivando o uso de máscara integralmente e medidas de higiene frequentes. Reforçado a importância de evitar visitas durante o puerpério, tanto pela fragilidade do RN que ainda não tomou as vacinas preconizadas, quanto para evitar a propagação do coronavírus.

Em razão desses cuidados, as consultas de puericultura não estavam sendo marcadas. Porém essa não era a medida correta, pois muitos bebês e crianças estavam ficando desassistidos em um momento delicado que se encontravam mais tempo em casa, expostas a diferentes riscos, sendo preciso investigação por parte dos profissionais. Dessa forma as consultas começaram a ser agendadas em menor quantidade, e assim começou os atendimentos de maneira a investigar o bem-estar das crianças e prestar todos os cuidados necessários, evitando o surgimento de acidentes infantis.

Por razão da pandemia, as crianças passam o tempo inteiramente no ambiente familiar, não sendo cuidadas muitas vezes pelos pais, devido suas ocupações no mercado de trabalho, ou mesmo que sejam por eles também estão passíveis de distração e cansaço físico e mental. Portanto para promover cuidados às crianças é fundamental alertar pais e cuidadores sobre a prevenção de acidentes infantis, pois o número de acidentes tem aumentado durante a pandemia. Medidas simples podem ser realizadas, como: evitar água em recipientes como baldes e caixas em fácil acesso, panelas com cabo para trás evitando queimaduras, supervisão na hora da alimentação, tomadas com proteção, entre outras, além de atenção à saúde mental dos pequenos, procurando dar atenção e carinho aos mesmos, trabalhando com jogos e brincadeiras criativas. Dessa forma, a ESF precisa transmitir essas informações à população (Brasil, 2020b; Programa de Apoio ao Desenvolvimento Infantil [PADIN], 2020).

As consultas de planejamento sexual e reprodutivo aconteciam normalmente, sendo observado que apenas mulheres procuram por esse atendimento, também não foi notório consultas de pré-concepção, muitas vezes o objetivo era a escolha de métodos anticoncepcionais, sendo os mais procurados: injeção mensal de hormônios combinados, anticoncepcional oral combinado, e injeção trimestral de progesterona. A coleta de material para realização do exame citopatológico, com a finalidade de rastreamento do câncer do colo do útero aconteciam com agendamento prévio, assim como o Exame Clínico das Mamas (ECM) para rastreamento do câncer de mama. 
O exame citopatológico é indicado para mulheres de 25 a 64 anos de idade, que tenham vida sexual ativa, com intervalo de 03 anos entre os exames, depois de 02 resultados consecutivos normais (Instituto Nacional de Câncer José Alencar Gomes da Silva [INCA], 2016). Porém foi realizado o rastreamento em todas as mulheres com vida sexual ativa, sendo que a paciente mais nova que compareceu para realizar o exame era uma adolescente de 15 anos. Assim devido a disponibilidade dos acadêmicos em realizar o exame, seguiu-se a Lei $\mathrm{n}^{\circ} 11.664$ (2008) que garante o direito ao exame para todas as mulheres que tenham iniciado a vida sexual, independentemente da idade.

Realizou-se inclusive evento de educação em saúde sobre o autocuidado feminino, com restrição de pessoas e com distanciamento preconizado, fazendo também no momento, de maneira organizada, o ECM e a coleta de exame citopatológico do colo do útero, trabalhando dessa forma com inovação para superar as dificuldades impostas pelo coronavírus na prevenção de outras patologias.

As consultas de hiperdia sofreram diferenças, na primeira unidade de estágio as consultas foram suspensas durante todo o período, as pessoas iam no local apenas pegar a receita que era renovada pelo médico sem que houvesse contato com o paciente. Já na segunda unidade, as consultas de hiperdia eram realizadas com devido agendamento prévio, a fim de evitar aglomerações, pois a maioria eram idosos, portanto, grupo de risco. Fica evidente a importância do acompanhamento dos indivíduos, pois o estado de saúde pode mudar em curto período de tempo.

Durante essas consultas ficou evidente a dificuldade dos pacientes quanto a utilização de medicamentos, não fazendo um bom controle das patologias. Dois pacientes apresentavam Hipertensão Arterial Sistêmica (HAS), Diabetes mellitus (DM) e hipercolesterolemia, todos descompensados, em níveis altos, tinham baixa escolaridade, sendo desconhecedores das letras e dos números, dessa forma foi identificado uso errado de medicamentos devido ao desconhecimento das drogas e seus respectivos horários, havendo o uso incorreto.

O Uso Racional de Medicamentos (URM) engloba a prescrição apropriada, disponibilidade, consumo em doses, horários e intervalos corretos, evitando interação entre drogas, fazendo um acompanhamento seguro, eficaz, e de qualidade. Porém, o uso incorreto pode levar malefícios e situações adversas aos indivíduos, causando complicações no estado de saúde (Monteiro \& Lacerda, 2016).

Como intervenção para esses casos, foram comprados com recursos próprios, 03 vasinhos para cada paciente, que continham separação dos 07 dias da semana. Assim um vasinho ficou para a semana durante a manhã com a figura do sol, outro com os medicamentos a serem utilizados após o almoço com a figura do garfo e faca, e outro recipiente para o período da noite com a figura da lua e das estrelas. Dessa forma os comprimidos foram separados e colocados nas caixinhas de horário da dose, sendo que o paciente não precisava saber os dias da semana, apenas abrir uma caixinha e tomar o conteúdo dela, importante enfatizar que os comprimidos não foram tirados de sua proteção, foram separados de maneira a continuar embalados. Assim havia reposição semanal das caixinhas e medição diária da pressão arterial e da glicemia em jejum, sendo alcançado resultados satisfatórios.

As pessoas com Doenças Crônicas Não Transmissíveis (DCNT) precisam de atenção especial nesse contexto de pandemia, pois apresentam duplo risco em seu estado de saúde. À vista disso é preciso a realização da estratificação de risco, para dessa maneira direcionar o atendimento adequado para o contexto de cada cidadão, pode ser realizado teleatendimento ou o estabelecimento de rotina de consultas da forma mais adequada. É preciso levar em consideração que as pessoas podem diminuir a adesão terapêutica devido a diminuição do contato com profissionais de saúde, assim os agentes de saúde são extremamente importantes nesse processo de acompanhamento (Brasil, 2020c).

Os ACS são parte fundamental para o bom funcionamento de uma ESF, eles também precisaram reinventar a forma de trabalhar. A assistência dos ACS não parou, algo que auxilia é a utilização de aplicativos de mensagem, com criação de 
grupos para a transmissão de informações, além disso as visitas têm voltado de maneira gradual, em casos de necessidade, com todas as medidas de precaução.

Os profissionais de saúde passam constantemente por situações que abalam a saúde mental dos mesmos, devido à sobrecarga e conflitos frente a situações que fogem do controle da ESF, havendo o medo diário de transmitir o vírus para as pessoas do convívio familiar. Os enfermeiros não estão livres dessas situações, a pandemia fez com que esse profissional atuante nesse setor fique totalmente sobrecarregado ao ter que agir na parte do gerenciamento de toda a unidade, realizar atendimentos, pois o financiamento desse estabelecimento de saúde é por produção constada no sistema, além de atuar na sala de vacina, devido a vacinação da Covid-19 ter um processo rígido de supervisão e controle.

Pesquisa realizada pela Fiocruz, em território nacional, revela que a pandemia afetou de maneira significativa a vida de 95\% dos trabalhadores da saúde, apontando ainda que 50\% referem sobrecarga, com carga de horário excessiva e exaustiva. Em complexidade ainda mostra que 43,2\% dos trabalhadores não se sentem seguros e protegidos para o enfrentamento da pandemia, sobretudo devido a falta de EPIs, além de identificar graves consequências na saúde mental dos profissionais, sendo comum perturbação do sono, irritabilidade e choro frequente (Leonel, 2021).

O estágio teve início em um momento crítico na pandemia, inclusive com alto número de leitos ocupados nos hospitais, dessa forma, pacientes sintomáticos respiratórios foram orientados a procurar atendimento na ESF, culminando no aumento de risco para pacientes que iam à unidade a procura de atendimento de rotina e também para os profissionais. Assim, entende-se que a pandemia levou profundas transformações na vida de todas as pessoas, sobretudo na ESF que além de agir na prevenção, promoção, reabilitação e controle do estado de saúde, foi preciso intervir em casos de Covid-19 para os quais não estavam preparados.

\section{Considerações Finais}

Dessa forma, compreende-se que o Estágio Supervisionado I proporciona muito conhecimento para o acadêmico de enfermagem, ao conciliar aprendizado técnico com o aprendizado intelectual e sentimental. Durante a pandemia o sentimento de medo e insegurança muitas vezes se fizeram presentes, mas serviram para fortalecer os futuros enfermeiros, e também para sensibilizar a população quanto a importância da enfermagem para o bom funcionamento de todos os serviços de saúde.

Assim, o objetivo de relatar sobre a experiência de estágio na ESF, durante o período de pandemia, foi cumprido, ressaltando que os serviços de saúde não estão preparados para o momento de inúmeras mudanças em curto espaço de tempo, pois alguns programas estavam em segundo plano, portanto a população ficava desassistida. No entanto, esse estudo possui como limitação o curto período de estágio e a vivência de campo em apenas uma cidade.

Reflete-se então sobre a importância do fortalecimento da ESF, enfatizando e valorizando os serviços de prevenção, promoção e recuperação da saúde oferecidos na mesma, pois a Atenção Básica precisa ser sólida para ser porta de entrada para os outros serviços de saúde, de forma a não sobrecarregar os outros níveis de atenção, sobretudo o setor terciário que passa por um momento delicado durante a pandemia. Por conseguinte, a pandemia está gerando mudanças e redimensionamento na prestação dos cuidados, mas não diminuiu a qualidade do atendimento, sobretudo devido ao trabalho em equipe de maneira qualificada e comprometida com o bem-estar humano.

Sugere-se que em trabalhos futuros os autores enfatizem a importância dos discentes de enfermagem serem inseridos precocemente no campo de estágio, bem como ressaltar a importância dos enfermeiros no enfrentamento da covid-19, trabalhando as limitações e medidas de superação. 


\section{Referências}

Agência Nacional de Vigilância Sanitária. (2021). Orientações para Serviços de Saúde: Medidas de prevenção e controle que devem ser adotadas durante a assistência aos casos suspeitos ou confirmados de infecção pelo novo coronavírus (SARS-cov-2).

Brasil. Ministério da Saúde. (2017). Política Nacional de Atenção Básica. Brasília: Ministério da Saúde.

Brasil. Ministério da Saúde. (2020a). Protocolo de manejo clínico do coronavírus (Covid-19) na atenção primária à saude. Versão 9. Brasília: Ministério da Saúde.

Brasil. Ministério da Saúde. (2020b). Crianças na pandemia Covid 19. Brasília. Ministério da Saúde.

Brasil. Ministério da Saúde. (2020c). Secretaria de Atenção Primária à Saúde. Departamento de Atenção à Saúde. Como organizar o cuidado de pessoas com doenças crônicas na APS no contexto de pandemia. Brasília. Ministério da Saúde.

Cavalcante, E. F. O. Pereira, L. R. B. O., Leite, M. J. V. F., Santos, A. M. D. \& Cavalcante, C. A. A. (2019). Implementação dos núcleos de segurança do paciente e as infecções relacionadas à assistência à saúde. Rev. Gaúcha Enferm. Rio Grande do Sul, v.40, e20180306. doi: 10.1590/1983-1447.2019.20180306

Cunha, A. L. A., Cornejo, A. A. Q., Hilari, A. A., Cayoja, A.V., Mendoza, J. M. C. \& Carrasco, O. V. (2020). Breve historia y fisiopatología del covid-19. Cuad. - Hosp. Clín., La Paz. 61(1), 130-43. http://www.scielo.org.bo/scielo.php?script=sci_arttext\&pid=S1652-67762020000100011

Constituição da República Federativa do Brasil. (2008). Lei nº 11.664. Brasília.

Cruz, C. S., Nunes, P. H. V., França, W. W. M., Rocha, J. V. R., Batista, J. J., Aguiar, T. W. A., Silva, A. M., Diniz, E. G. M., Aires, A. L., Cabral, L. P., Silva, A. L., Silva, H. A. M. F., Araújo, H. D. A., Albuquerque, M. C. P. A. \& Aires, A. L. (2021). Protagonismo do estudante universitário na prevenção da infecção pelo Trichomonas vaginalis. Research, Society and Development. 10(7), e20010715345. doi: 10.33448/rsd- v10i7. 15345

Estrela, F. M., Silva, K. K. A., Cruz, M. A., \& Gomes, N. P. (2020). Gestantes no contexto da pandemia da Covid-19: reflexões e desafios. Physis: Revista de Saúde Coletiva [online]. v. 30, e300215. doi: 10.1590/S0103-73312020300215

Gil, A. C. (2008). Como elaborar projetos de pesquisa. São Paulo: Atlas.

Giovanella, L. Bousquat, A., Schenkman, S., Almeida, P. F., Sardinha, L. M. V. \& Vieira, M. L. F. V. (2021). Cobertura da Estratégia Saúde da Família no Brasil: o que nos mostram as Pesquisas Nacionais de Saúde 2013 e 2019. Ciência \& Saúde Coletiva [online]. v. 26, 2543-2556. doi: 10.1590/141381232021266.1 .43952020

Instituto Nacional de Câncer José Alencar Gomes da Silva. (2016). Diretrizes Brasileiras para o Rastreamento do Câncer do Colo do Útero. 2 ed. Rio de Janeiro.

Leonel, F. (2021). Fiocruz. Pesquisa analisa o impacto da pandemia entre profissionais de saúde. https://portal.fiocruz.br/noticia/pesquisa-analisa-o-impactoda-pandemia-entre-profissionais-de-saude

Lima, F. A. \& Lima, S. C. (2020). Construindo cidades saudáveis: a instrumentalização de políticas públicas intersetoriais de saúde a partir do Planejamento Estratégico Situacional. Saúde soc. São Paulo, v. 29, e200058. doi: 10.1590/s0104-12902020200058

Marques, R. \& Fraguas, T. (2021). A formação do senso crítico no processo de ensino e aprendizagem como forma de superação do senso comum. Research, Society and Development, v. 10, e31010716655. doi: 10.33448/rsd-v10i7.16655

Monteiro, E. R. \& Lacerda, J. T. (2016). Promoção do uso racional de medicamentos: uma proposta de modelo avaliativo da gestão municipal. Saúde Debate. Rio de Janeiro, v. 40, 101-116. doi: 10.1590/0103-1104201611108.

Programa de Apoio ao Desenvolvimento Infantil. (2020). $3^{\text {a }}$ Cartilha de Orientação para desenvolvimento do PADIN no período de desenvolvimento social. Ceará.

Rigobello, J. L., Bernardes. A., Moura, A. A., Zanetti, A. C. B., Spiri, W. C., \& Gabriel, C. S. (2018). Supervised Curricular Internship and the development of management skills: a perception of graduates, undergraduates, and professors. Escola Anna Nery [online]. v. 22. doi:10.1590/2177-9465-EAN-2017-0298

Silva, I. M., Silva, M. T. B. F., Santos, R. G. \& Ferreira, R. K. G. (2021)._Trabalho da Equipe Multiprofissional no contexto da COVID-19: Diversos olhares, um só objetivo. Research, Society and Development, v. 10, e53210313439. doi: http://dx.doi.org/10.33448/rsd-v10i3.13439

Tesini, B. L. (2021). Coronavírus e síndromes respiratórias agudas (covid-19, mers e sars). Ministério da Saúde https://www.msdmanuals.com/pt/profissional/doen\%C3\%A7as-infecciosas/v\%C3\%ADrus-respirat\%C3\%B3rios/coronav\%C3\%ADrus-e-s\%C3\%ADndromesrespirat\%C3\%B3rias-agudas-covid-19-mers-e-sars 